\title{
Autoevaluación de formación en habilidades de entrevista, relación médico paciente y comunicación en médicos graduados
}

\author{
Cecilia Sogi ${ }^{1}$, Salomón Zavala ${ }^{1}$, Miguel Oliveros ${ }^{1}$, Carlos Salcedo ${ }^{1}$
}

Resumen

Palabras clave

\begin{abstract}
Objetivo: Evaluar la percepción de graduados de medicina sobre su formación en habilidades de entrevista, relación médico paciente y comunicación. Materiales y Métodos: La población de estudio fue postulantes a plazas de residente de la Universidad Nacional Mayor de San Marcos, para el año 2005. El cuestionario utilizado fue una adaptación de tres instrumentos, sometidos previamente a estudios de validez y confiabilidad. Resultados: Participaron 917 graduados, $59 \%$ varones, $56 \%$ entre los 25 y 29 años de edad. Pese a que el $85 \%$ respondió haber tenido formación en habilidades clínicas básicas, se identificó deficiencias en el dominio de: a) el método clínico; b) la obtención de información psicosocial; c) la evaluación de la comprensión del paciente; d) el manejo de emociones; e) la comunicación no verbal. En comentarios de los participantes, se identificó el factor tiempo como limitante para la adecuada relación/comunicación con el paciente. Conclusiones: Se identificó deficiencias en habilidades clínicas básicas, a ser considerado en programas de intervención educativa.
\end{abstract}

Entrevistas; relaciones médico-paciente; educación médica; comunicación.
Self-assessment of core clinical skills in medical graduates: medical interview, physician patient relationship and communication

\section{Abstract}

Objective: To assess the perception of medical graduates in regards to their training in medical interview, physician-patient relationship and communication skills. Materials and Methods: Applicants to a 2005-residency post at the Universidad Nacional Mayor de San Marcos participated in the study. The questionnaire was designed adapting three instruments. Validity and reliability studies were carried out. Results: A total of 917 graduates completed the questionnaire, $59 \%$ were male; $56 \%$ between 25 -29years old; $85 \%$ responded to having had training in core clinical skill, but various deficiencies were identified: a) clinical method; $b$ ) psychosocial data gathering; c) patient's comprehension examination; d) emotions management; e) non verbal communication. The time as limiting factor for adequate relationship/communication with the patient was identified in respondents' comments. Conclusion: Several deficiencies in core clinical skills were identified and should be considered in educational intervention programs.

1 Facultad de Medicina, UNMSM. Lima, Perú.
Keywords: Interviews; physician-patient relations; education, medical; communication.

\section{INTRODUCCIÓN}

Se considera habilidades clínicas esenciales de todo médico la entrevista, la relación médico paciente y la comunicación. Estas tres habilidades, aunque tratadas por separado, están imbricadas. La entrevista es el medio para establecer la relación y comunicación con el paciente y éstas, a su vez, influyen en la calidad de la primera.

Entrevista médica. Prueba de que la entrevista es la principal habilidad en medicina es que "por sí sola produce los datos necesarios para el diagnóstico, más de las tres cuartas partes de las veces; y establece más diagnósticos que los datos del examen físico y de laboratorio combinados. Genera más datos necesarios para tratamiento y prevención, y es el 
principal medio para transmitir información al paciente" $\left({ }^{1}\right)$.

En un editorial de Annals of Internal Medicine, Duffy dice: "La entrevista médica contemporánea es un diálogo terapéutico entre médico y paciente; es el principal método diagnóstico y componente esencial de toda terapia. Sin ese diálogo, generalmente la terapia fracasa, especialmente en enfermedades que se manifiestan por somatización” ${ }^{(2)}$.

La importancia de la entrevista ha sido reconocida especialmente en atención primaria. Se estima que un médico general lleva a cabo, en promedio, entre $120000 \mathrm{y}$ 160000 entrevistas durante una carrera profesional de 40 años $\left(^{3}\right)$.

Relación médico paciente. La interacción fundamental en la atención de salud es aquella que se realiza entre el paciente y el médico. Es más, este hecho es probable que continúe siendo así en un futuro previsible $\left({ }^{4}\right)$. En otras palabras, Siegler (citado por Chin) dice: "pese a los notables desarrollos científicos del último siglo, la relación médico paciente ha sido descrita como un acontecimiento inmutable en medicina, que se preserva principalmente por las metas inmutables de la medicina" $\left({ }^{5}\right)$.

De otro lado, la frase pacto de confianza (covenant of trust), acuñada por la American Medical Association caracteriza lo esencial de la relación médico paciente. Esta relación es inherentemente una acción benevolente, en la que una persona pone su vida en manos de otra, un acto de notable confianza, esperanza y respeto. Confianza, que a su vez, se basa en la creencia de que el médico lo tratará buscando su mejor interés $\left(^{6}\right)$.

Chin agrega que "la confianza es una de las fuerzas más vitales que sostiene toda relación exitosa. La vulnerabilidad de los pacientes y su necesidad de atención los fuerza a confiar en los médicos. Los pacientes ven la confianza generalmente como un proceso interactivo, necesidad de atención, interés y compasión, y esencialmente de ser escuchado" $\left({ }^{5}\right)$.

Centralidad del paciente. Hoy en día, se considera que la mejor relación médico paciente es aquella enfocada en este último. La atención centrada en el paciente es definida como congruente con y sensible a las necesidades y preferencias del paciente. Esta idea ha sido un componente del movimiento de los derechos del paciente desde los años sesenta. Una revisión del tema señala que la evidencia tangible del citado fenómeno está presente ahora en todas las facetas de la medicina; en la información al paciente, la decisión médica, la legislación concerniente al consentimiento informado y las decisiones sobre tratamientos de soporte vital; así como la perspectiva del paciente en la formación médica, investigación y evaluación de calidad de los servicios de salud $\left({ }^{7}\right)$.

En una revisión del concepto centrado en el paciente, Bensing y colaboradores dicen: "El consenso de los estudios es que el centrado en el paciente implica sentar el diálogo terapéutico más en un paradigma psicosocial que biomédico, con compromiso activo del paciente en ese diálogo, siendo el médico abierto y sensible a la agenda y perspectivas del paciente, incluyendo evocar intereses, expectativas y preferencias de tratamiento, y establecer la relación emocional" $\left({ }^{8}\right)$.

Comunicación. La declaración de consenso (Toronto Consensus Statement) publicada por el British Medical Journal, en 1991, dice: "La comunicación médico paciente efectiva es una función clínica esencial que no puede ser delegada" $\left({ }^{9}\right)$.

Caracteriza lo esencial de la práctica médica la comunicación verbal y no verbal que se establece en el encuentro con el paciente, dicen Travaline y colaboradores. 
Agregan que, además de compartir información diagnóstica y terapéutica, la mayoría de médicos reconocerá que estos encuentros involucran también la búsqueda del paciente de salud psicológica, conexión o relación terapéutica $\left({ }^{10}\right)$.

El informe Kalamazoo II vincula la comunicación efectiva a: a) una mayor satisfacción del médico y el paciente; b) adherencia a los planes de tratamiento; c) decisiones médicas más adecuadas; d) mejores resultados de salud; e) disminución en la incidencia de demandas por mala práctica contra médicos de atención primaria; f) información y educación del público $\left({ }^{11}\right)$.

En resumen, la revisión de la literatura señala que las habilidades clínicas de entrevista, relación médico paciente y comunicación influyen en los resultados de la atención de salud del paciente, a corto, mediano y largo plazo. Y para ello resultan esenciales la empatía, conducta centrada en el paciente, dar aliento y también liberar la tensión $\left(^{3}\right)$. En otras palabras, como señalan Travaline y colaboradores, los resultados de salud influenciados por la comunicación incluyen: salud emocional, resolución de síntomas, capacidad funcional, alivio del dolor y medidas fisiológicas, tales como niveles de presión arterial y glicemia $\left({ }^{10}\right)$.

Educación médica. Makoul señala que la enseñanza y la evaluación de las habilidades de comunicación han llegado a ser visibles en la educación médica en los Estados Unidos. Tales habilidades se destacan de manera prominente en una nueva iniciativa nacional: the National Board of Medical Examiners, the Federation of State Medical Boards, y the Educational Commission for Foreign Medical Graduates están trabajando juntos para implementar un examen de habilidades clínicas utilizando pacientes estandarizados, a ser administrados en el tercer y cuarto año de medicina, como parte del United States Medical Licensing
Examination (USMLE). Este examen "requiere que los estudiantes demuestren que pueden obtener información de los pacientes, llevar a cabo el examen físico y comunicar sus hallazgos a pacientes y colegas" $\left({ }^{12}\right)$.

Objetivos del estudio. El presente estudio es una primera aproximación al tema de evaluación de las habilidades clínicas básicas: entrevista, relación médico paciente y comunicación. Se basa en el análisis de la forma como los médicos graduados perciben su formación en las mencionadas habilidades.

\section{MATERIALES Y MÉTODOS}

La población de estudio estuvo conformada por médicos graduados, postulantes a las plazas de residencia de la Universidad Nacional Mayor de San Marcos (UNMSM). El total de inscritos en el proceso de admisión 2005 fueron 1410 y completaron el cuestionario de habilidades clínicas 947 (67\%).

El cuestionario de habilidades clínicas es una adaptación de tres instrumentos: 1) SEGUE Framework for Teaching and Assessing Communication Skills, AAMC $\left({ }^{13}\right)$, una lista de chequeo de las tareas de comunicación en medicina; 2) Structured Clinical Observation de Lane y Gottlieb $\left({ }^{14}\right)$; 3) Physician Communication Skills de Ashbury, Iverson y Kralj $\left({ }^{15}\right)$.

El cuestionario en mención obtiene datos sobre: a) inicio de la entrevista, b) obtención de información, c) información al paciente, d) comprensión del paciente, e) habilidades de relación, f) modales del médico, g) término del encuentro. Adicionalmente, recoge datos sociodemográficos (edad, sexo, año de graduación, universidad y si tuvo formación en habilidades clínicas).

La validez y confiabilidad del instrumento fueron determinadas a través de un estudio 
piloto en el que participaron 30 residentes de medicina intensiva.

La validez del contenido fue determinada mediante la prueba de jueces. Participaron cinco médicos docentes de diversas especialidades (medicina interna, pediatría, neurología y psiquiatría). Completaron un cuestionario de preguntas sobre el instrumento y los datos fueron analizados para examinar la concordancia entre evaluadores. En cada pregunta se utilizó la prueba binomial, considerándose significativa la concordancia $p<0,05$. En ocho de las nueve preguntas, la concordancia interevaluadores fue de $p<0,05$.

La validez interna fue determinada mediante el método del item test, que consiste en correlacionar cada item con el puntaje total (coeficiente de Pearson). Se consideró válidos los ítems cuyo $r$ de Pearson fueron iguales o mayores que 0,20. En los resultados del item test seis preguntas tuvieron $\mathrm{r}<0,20$, las mismas que fueron examinadas siguiendo las recomendaciones de Garret $\left({ }^{16}\right)$. Se eliminó dos preguntas y en la versión final del cuestionario de habilidades clínicas quedaron 52 .

Se determinó la confiabilidad del instrumento mediante el coeficiente alfa de Cronbach, la misma que fue de 0,9518.

Se solicitó autorización para la aplicación del cuestionario a la Dirección de la Unidad de Postgrado de la Facultad de Medicina de la UNMSM, así como a la Comisión a cargo del proceso de admisión.

Los datos fueron recogidos en el proceso de inscripción de los postulantes, el mismo que tuvo lugar en marzo de 2005. Dos personas entrenadas estuvieron a cargo del trabajo de campo, supervisadas por los investigadores.

Concluido el trabajo de campo, los cuestionarios fueron revisados, separándose aquellos incompletos, los que marcaron todas las respuestas en una sola columna y los que respondieron algunas veces (3) en la pregunta 53 ¿He respondido sincera y honestamente a las frases anteriores? En este proceso se separó 30 cuestionarios $(3 \%$ de 947), los mismos que fueron descartados, quedando 917 en la base de datos.

Con relación a la ética, se explicó que la participación era voluntaria y que los datos serían utilizados solo para los propósitos de la investigación.

El procesamiento y análisis de datos se realizó usando los paquetes estadísticos SPSS 9,0 para Windows y EpiInfo 6,0 para DOS. Elaborada la base de datos en Excel, se procedió a hacer tablas con una y dos variables. El análisis de datos cualitativos se realizó utilizando el Atlas ti.

La escala de estanones fue utilizada para categorizar los sujetos de estudio en tres grupos (habilidades buenas, regulares y malas), según los puntajes parciales y el puntaje total obtenido en el cuestionario. Se obtuvo la media aritmética (x) y la desviación estándar (s) de los puntajes con cuyos resultados se calculó los puntos de corte: $\mathrm{a}=\mathrm{x}-0,75$ (s) $\mathrm{y} \mathrm{b}=\mathrm{x}+0,75$ (s).

\section{RESULTADOS}

Respondieron el cuestionario las dos terceras partes de los postulantes inscritos en el proceso de admisión 2005 a plazas de residencia en la UNMSM. Los no participantes fueron, en su mayoría, personas que mediante poder inscribieron al postulante y por consiguiente no se les administró el cuestionario.

Del total de participantes en el estudio $(\mathrm{n}=917), 58,8 \%$ fueron varones y más de la mitad $(55,8 \%)$ tenía entre 25 y 29 años, seguidos de aquellos entre los 30 y 34 años (31\%). La edad media de los varones fue 
30,3 (DE 4,8) y en las mujeres 29,0 (DE $3,7)$; la edad mínima fue 23 años y la máxima 66 .

El $85 \%$ respondió haber tenido formación en las habilidades que nos ocupa, en cursos de los más diversos.

La Tabla 1 presenta la suma de frecuencias de las respuestas nunca (1), casi nunca (2) y algunas veces (3), consideradas habilidades deficientes

El uso de preguntas directivas y múltiples en la entrevista estaría relacionado con deficiencias en el dominio del método clínico. El no pedir la opinión del paciente acerca de su problema y como éstos afectaron su vida indicarían una aproximación más biomédica que psicosocial en la entrevista. En información al paciente, la cuarta parte

Tabla 1. Habilidades clínicas deficientes.

\begin{tabular}{lr}
\hline Respuestas & $\begin{array}{c}\text { Habilidades } \\
\text { 1, } 2 \text { y } 3 \%\end{array}$ \\
\hline Obtiene información & \\
Evita preguntas directivas & 49,1 \\
Pide opinión del paciente acerca de su problema & 46,9 \\
Pregunta como los problemas de salud afectan su vida & 33,6 \\
Evita preguntas múltiples & 33,4 \\
Información al paciente & \\
Alienta al paciente a hacer preguntas & 31,7 \\
Evalúa habilidad del paciente para seguir indicaciones & 26,0 \\
Evalúa disposición del paciente a seguir indicaciones & 24,1 \\
Evalúa nivel de comprensión del paciente & 21,4 \\
Comprensión del paciente & \\
Identifica y sigue señales no verbales & 28,2 \\
Reconoce tiempo de espera del paciente & 20,0 \\
Habilidades de relación & \\
Alienta exprese emociones relacionadas con & 21,3 \\
problema actual & 20,0 \\
Identifica necesidades psicológicas/emocionales & \\
Modales del médico & 28,8 \\
Usa adecuadamente el silencio & 21,1 \\
Usa adecuadamente la mímica facial & \\
Término del encuentro & 36,0 \\
Resume temas importantes & 26,3 \\
Revisa los pasos siguientes & 25,2 \\
Informa que la entrevista está terminando & 20,4 \\
Asegura compromiso del paciente a seguir tratamiento & \\
\hline
\end{tabular}

Tabla 2. Puntajes globales en habilidades clínicas.

\begin{tabular}{lccc}
\hline & $\begin{array}{c}\text { Bajo } \\
\%\end{array}$ & $\begin{array}{c}\text { Medio } \\
\%\end{array}$ & $\begin{array}{c}\text { Alto } \\
\%\end{array}$ \\
\hline Puntaje total & 22,1 & 53,2 & 24,6 \\
Inicia sesión de entrevista & 31,6 & 48,1 & 20,3 \\
Obtiene información & 27,2 & 51,1 & 21,7 \\
Información al paciente & 26,6 & 51,3 & 22,1 \\
Comprensión del paciente & 27,3 & 56,7 & 16,0 \\
Habilidades de relación & 26,4 & 48,1 & 25,5 \\
Modales del médico & 27,4 & 55,0 & 17,7 \\
Término de la entrevista & 22,0 & 56,4 & 21,6 \\
\hline
\end{tabular}

de los encuestados no evalúa capacidad mental o competencia del paciente. El no alentar la expresión de emociones y no identificar necesidades psicológicas indicarían deficiencias en el manejo de las emociones. La no identificación de señales no verbales indicaría deficiencias en las habilidades de comunicación no verbal.

La Tabla 2 presenta los puntajes globales en cada sección del cuestionario obtenidos en la escala de estanones. Los puntajes más bajos por cada sección del cuestionario fueron: inicia sesión de entrevista, modales del médico, comprensión del paciente y obtiene información.

El cuestionario utilizado proporciona un espacio para comentarios. Uno de cada cinco participantes en el estudio (21,3\% de 917) hizo comentarios y éstos fueron clasificados en cinco categorías. El 15,9\% de todos los comentarios hizo mención al factor tiempo. A continuación se presenta ejemplos:

- Usualmente hay poco tiempo para explicarles con el detalle que uno desea.

- No es posible realizarlo de la forma ideal por la falta de tiempo y la entrevista tiene que ser dirigida al motivo de la consulta.

- A veces, por la presión del número de pacientes que tengo que atender obligatoriamente, no puedo, por el tiempo, realizar preguntas para ver si el paciente entendió todo, así como otras preguntas 
que están en este cuestionario. Por eso, trato de escribir todo en la receta o hablar claro.

- La actitud frente al paciente está también influenciada por factores externos, como necesidad del médico de terminar de atender sus 20 a 24 pacientes.

- Es de presumir que una entrevista con estas características lleva por lo menos 30 minutos, por lo que personalmente he tenido problemas y quejas por la demora de la consulta.

\section{DISCUSIÓN}

Consideramos que la principal limitación del estudio es que los datos fueron obtenidos a través de un cuestionario autoadministrado. Aunque este es el mejor método para obtener datos sobre actitudes y autoeficacia, como señalan Ashbury y colaboradores, el método de la observación es un procedimiento más riguroso para obtener datos reales sobre el uso de estrategias de comunicación $\left({ }^{15}\right)$. Coincidimos con los autores citados que parece razonable asumir que los datos conductuales sobreestiman el uso real de esas habilidades. Otra limitación es el tipo de cuestionario utilizado, que, como se mencionó en la sección correspondiente, es una adaptación de tres instrumentos. Esto hace que nuestros resultados no sean estrictamente comparables con los de otros autores.

No obstante las limitaciones señaladas, nuestros datos corresponden a 917 graduados de la mayoría de las universidades públicas y privadas nacionales y algunas extranjeras. Esto permite una primera aproximación al tema de formación en habilidades clínicas básicas; asimismo, el conocimiento existente permite una discusión adecuada de nuestro hallazgo.

En nuestro estudio, pese a que el $85 \%$ respondió haber tenido formación en habilidades clínicas básicas, se identificó deficiencias en: a) método clínico, b) obtención de información psicosocial, c) evaluación de competencia en la información al paciente, d) comunicación no verbal, e) manejo de emociones y f) término del encuentro.

Respecto al método clínico en el proceso diagnóstico, nuestro hallazgo es similar al de Moreno Rodríguez, pese a diferencias metodológicas. El autor llevó a cabo un estudio en internos, bajo observación directa: "las deficiencias más frecuentes de las mayores fue el uso de preguntas que inducen respuesta, un tipo de pregunta cerrada y terminal, formulada medio afirmativa, medio interrogativamente, en forma positiva o negativa, casi siempre muy capciosa, que exige de sí o no $(58,3 \%)$ y obedece a que desde el comienzo el alumno está construyendo el diagnóstico en su mente e inadvertidamente pregunta lo que desearía escuchar del paciente...comenzar el interrogatorio por los antecedentes patológicos y obtener la información por preguntas, limitando la expresión abierta del enfermo, fue una característica de numerosas entrevistas... excepcionalmente abordaron aspectos psicológicos y sociales, incluso si el enfermo los expresó claramente" $\left({ }^{17}\right)$.

En nuestro estudio, las deficiencias en obtención de información psicosocial y la no evaluación de la comprensión del paciente estaría indicando un modelo de relación médico paciente paternalista. En este modelo, el médico, como tutor del paciente, toma la decisión por éste, asume que los valores del paciente son consistentes con los de él y la autonomía del paciente se reduce a consentir con las recomendaciones del médico $\left({ }^{18}\right)$.

Respecto al manejo de las emociones, Suchman y colaboradores señalan que "la habilidad empática básica parece ser reconocer cuándo están presentes las emociones, aunque no sean expresadas 
directamente, invitando a explorarlas y reconocerlas efectivamente, de modo que el paciente se sienta comprendido" $\left({ }^{19}\right)$. Por su lado, Buckman dice que la respuesta empática es una técnica que consiste en tres pasos: 1) identificar la emoción; 2) identificar la fuente de la emoción; 3) responder de manera que demuestre al paciente que uno ha establecido la conexión entre los dos pasos previos $\left({ }^{20}\right)$.

Maguire y Pitceathly hallaron las siguientes deficiencias en habilidades de comunicación de los médicos: es probable que obtengan solamente la mitad de las quejas y preocupaciones del paciente; que obtengan poca información sobre la percepción del paciente acerca de su problema o su impacto físico, emocional y social $\left({ }^{21}\right)$.

Nuestro hallazgo de que hay deficiencias en identificar señales no verbales, necesidades psicológicas del paciente y en asegurar el compromiso del paciente en adherirse al plan de tratamiento acordado, coincide con los de Ashbury y colaboradores en su encuesta a médicos generales y de familia en Newfoundland $\left({ }^{15}\right)$.

En nuestro estudio, el factor tiempo fue mencionado como una limitante para la comunicación eficaz y establecer la buena relación con el paciente; algunos mencionaron que el tiempo de consulta por paciente es de 10 minutos.

Respecto al tiempo, Benzing y colaboradores en su revisión hallaron, por un lado, que los médicos y los pacientes creen que la duración de la consulta es una dimensión importante de calidad de la atención y, por otro, muchos investigadores sostienen que el médico como persona y su estilo de trabajo tienen más impacto en la calidad de la atención que el tiempo de consulta. El aspecto del estilo de trabajo vinculado a calidad del servicio está comprendido en el amplio concepto de la atención centrada en el paciente. Los autores citados compararon consultas americanas y danesas en pacientes con hipertensión arterial. Hallaron que las primeras fueron 6 minutos más largas que las segundas $(15,4$ vs 9,5 minutos, respectivamente). Adicionalmente, hallaron que en las americanas el examen físico era más largo que en las danesas. Los médicos americanos hacen más preguntas, tanto de naturaleza psicosocial como biomédica; mientras que, los daneses era más probable que emplearan el intercambio afectivo, particularmente aquel asociado al establecimiento de la relación. Los pacientes americanos fueron diferentes de los daneses. El tipo de consulta más frecuente en los americanos (48\%) se caracterizó por contenido intensamente biomédico; mientras que el más frecuente en los daneses $(50 \%)$ se caracterizó por un contenido socio-emocional relativamente alto. Los autores atribuyen las diferencias descritas a la formación médica y filosófica, al sistema sanitario y a los valores y expectativas culturales relevantes en la atención de salud en cada país $\left({ }^{8}\right)$.

En conclusión, pese a que $85 \%$ de los médicos graduados respondió haber tenido formación en habilidades de entrevista, relación médico paciente y comunicación, se identificó deficiencias en el dominio de: a) el método clínico, b) la obtención de información psicosocial, c) la evaluación de la comprensión del paciente, d) el manejo de las emociones y f) la comunicación no verbal.

\section{A GRADECIMIENTO}

Estudio de Investigación 2005 Con Asignación a la Investigación y Con Incentivo al Investigador.

\section{REFERENCIAS BIBLIOGRÁFICAS}

1. Smith RC, Lyles JS, Mettler J, Stoffelmayr BE, Van Egeren LF, Marshall AA, et al. The effectiveness of intensive training for residents in interviewing. A randomized, controlled study. Ann Intern Med. 1998;128(2):118-26. 
2. Duffy FD. Dialogue. The core clinical skill [Editorial]. Ann Intern Med. 1998;128(2):139-41.

3. Beck RS, Daughtridge R, Sloane PD. Physician-patient communication in the primary care office: A systematic review. J Am Board Fam Pract. 2002;15:25-38.

4. American Healthways and John Hopkins. Defining the patientphysician relationship for the 21 st century. 3rd Annual Disease Management Outcomes Summit, October 30November 2, 2003. Phoenix, Arizona. 2004 American Healthways Inc.

5. Chin JJ. Doctor patient relationship: A covenant of trust. Singapore Med J. 2001:42(12):579-81.

6. Giorgianni SJ, Editor in Chief. The evolving patient-physician relationship. The Pfizer Journal. 1998;2(3).

7. Laine Ch, Davidoff F. Patient-centered medicine. A professional evolution. JAMA. 1996;275:152-6.

8. Bensing JM, Roter DL, Hulsman RL. Communication patterns of primary care physicians in the United States and the Netherlands. J Gen Intern Med. 2003;18:335-42.

9. Stewart M. Effective physician-patient communication and health outcomes: a review. CMAJ. 1995;152(9):1423-33.

10. Travaline JM, Ruchinskas R, D'Alonzo GE Jr. Patientphysician communication: Why and how. JAOA. 2005;105(1):13-8.

11. Duffy FD, Gordon GH, Whelan G, Cole-Kelly K, Frankel $\mathrm{R}$, All participants in the American Academy on Physician and Patient's Conference on Education and Evaluation of Competence in Communication and Interpersonal Skills. Assessing competence in communication and interpersonal skills: The Kalamazoo II Report. Acad Med. 2001;79:495507.

12. Makoul G. Communication skills education in medical school and beyond. JAMA. 2003;89:93.

13. Association of American Medical Colleges. Report III Contemporary Issues in Medicine: Communication in
Medicine. Medical School Objective Project. AAMC, October 1999.

14. Laine JL, Gottlieb RP. Structured clinical observation: A method to teach clinical skills with limited time and financial resources. Pediatrics. 2000;105(4):973-7.

15. Ashbury FD, Iverson DC, Kralj B. Physician communication skills: Results of a survey of general/family practitioners in Newfoundland. Med Educ Online (serial online). 2001;6:1. Disponible en: http://www.msu.edu/ dsolomon// res00014.pdf.

16. Garret HE. Estadística en psicología y educación. Barcelona: Editorial Paidos Ibérica; 1983.

17. Moreno-Rodríguez MA. Deficiencias en la entrevista médica. Un aspecto del método clínico. Rev Cubana Med. 2000;39(2):106-14.

18. Emanuel EJ, Emanuel LL. Four models of the physicianpatient relationship. JAMA. 1992;267:2221-6.

19. Suchman AL, Markakis K, Beckman HB, Frankel R. A model of empathic communication in the medical interview. JAMA. 1997;277:678-82.

20. Buckman R. Editorials. Communications and emotions. Skills and effort are key. BMJ. 2002;325:672.

21. Maguire P, Pitceathly C. Key communication skills and how to acquire them. BMJ. 2002;325:697-700.

Manuscrito recibido el 09 de octubre de 2005 y aceptado para publicación el 10 diciembre de 2005.

Correspondencia: Dra. Cecilia Sogi

Instituto de Ética en Salud

Facultad de Medicina, UNMSM.

Av Grau 755. Lima 1, Perú

Correo-e: ceciliasogi@yahoo.com 